\title{
PI3K rescues the detrimental effects of chronic Akt activation in the heart during ischemia/reperfusion injury
}

\author{
Tomohisa Nagoshi, ${ }^{1}$ Takashi Matsui, ${ }^{1}$ Takuma Aoyama, ${ }^{1}$ Annarosa Leri, ${ }^{2}$ Piero Anversa, ${ }^{2}$ \\ Ling Li, ${ }^{1}$ Wataru Ogawa, ${ }^{3}$ Federica del Monte, ${ }^{1}$ Judith K. Gwathmey, ${ }^{4,5}$ Luanda Grazette, ${ }^{1}$ \\ Brian Hemmings, ${ }^{6}$ David A. Kass,, ${ }^{7}$ Hunter C. Champion, ${ }^{7}$ and Anthony Rosenzweig ${ }^{1}$ \\ ${ }^{1}$ Program in Cardiovascular Gene Therapy, Cardiovascular Research Center, Massachusetts General Hospital, \\ Harvard Medical School, Boston, Massachusetts, USA. 2Department of Medicine, New York Medical College, Valhalla, New York, USA. \\ 3Department of Clinical Molecular Medicine, Division of Diabetes, Digestive, and Kidney Diseases, Kobe University Graduate School of Medicine, \\ Kobe, Japan. ${ }^{4}$ Gwathmey Inc., Cambridge, Massachusetts, USA. ${ }^{5}$ Harvard Medical School, Boston, Massachusetts, USA. ${ }^{6}$ Friedrich Miescher \\ Institute for Biomedical Research, Basel, Switzerland. Division of Cardiology, The Johns Hopkins Hospital, Baltimore, Maryland, USA.
}

\begin{abstract}
Acute activation of the serine-threonine kinase Akt is cardioprotective and reduces both infarction and dysfunction after ischemia/reperfusion injury (IRI). However, less is known about the chronic effects of Akt activation in the heart, and, paradoxically, Akt is activated in samples from patients with chronic heart failure. We generated $\mathrm{Tg}$ mice with cardiac-specific expression of either activated (myristoylated [myr]) or dominantnegative (dn) Akt and assessed their response to IRI in an ex vivo model. While dn-Akt hearts demonstrated a moderate reduction in functional recovery after IRI, no function was restored in any of the myr-Akt-Tg hearts. Moreover, infarcts were dramatically larger in myr-Akt-Tg hearts. Biochemical analyses demonstrated that chronic Akt activation induces feedback inhibition of PI3K activity through both proteasome-dependent degradation of insulin receptor substrate-1 (IRS-1) and inhibition of transcription of IRS-1 as well as that of IRS-2. To test the functional significance of these signaling changes, we performed in vivo cardiac gene transfer with constitutively active PI3K in myr-Akt-Tg mice. Restoration of PI3K rescued function and reduced injury after IRI. These data demonstrate that PI3K-dependent but Akt-independent effectors are required for full cardioprotection and suggest a mechanism by which chronic Akt activation can become maladaptive.
\end{abstract}

\section{Introduction}

Death of cardiomyocytes results in decreased cardiac function, which correlates with overall morbidity and mortality in many different clinical settings. For these reasons, understanding the intracellular signaling pathways that control cardiomyocyte survival has significant clinical implications.

Insulin and IGF-1 have beneficial effects on cardiomyocyte function and survival (1-6), including protection against acute ischemic injury $(5,7)$. Tg mice with cardiac-specific IGF-1 overexpression driven by the $\alpha$-myosin heavy chain ( $\alpha$-MHC) promoter (2) demonstrate less cardiomyocyte death and fibrosis with chronic coronary artery narrowing (6), as well as reduced injury in an ex vivo model of ischemia/reperfusion injury (IRI) (7). Activation of the serine-threonine kinase Akt is thought to play an important role in promoting cardiomyocyte survival in response to IGF-1 and similar ligands, and acute activation of Akt is cardioprotective in vitro and in vivo (8-10). However, little is known about the effects of chronic Akt activation, and, paradoxically, samples from

\footnotetext{
Nonstandard abbreviations used: Ad.myr-Akt, adenoviral myr-Akt; CPK, creatine phosphokinase; GSK3 $\beta$, glycogen synthase kinase-3 $\beta$; IRE, insulin response element; IRI, ischemia/reperfusion injury; IRS-1, insulin receptor substrate-1; LVDP, LV developed pressure; LVEDP, LV end-diastolic pressure; LVSP, LV systolic pressure; $\alpha$-MHC, $\alpha$-myosin heavy chain; myr-Akt, myristoylated Akt; mTOR, mammalian target of rapamycin; NRCM, neonatal rat cardiomyocyte; NTg, nontransgenic; PDK1, phosphoinositide-dependent kinase-1; QRT-PCR, quantitative RT-PCR; SGK1, serumand glucocorticoid-regulated protein kinase-1; TTC, triphenyltetrazolium chloride. Conflict of interest: The authors have declared that no conflict of interest exists.

humans with advanced heart failure demonstrate enhanced Akt phosphorylation (11), which suggests either that Akt activation is not sufficient for long-term cardioprotection or that it may actually have adverse effects over time.

Tyrosine kinase receptors, including the insulin and IGF-1 receptors, initiate signaling via the adaptor molecule insulin receptor substrate-1 (IRS-1), which docks with the SH2 domains of the p85 PI3K regulatory subunit (12). Genetic deletion of IRS-1 led to identification of IRS-2, an accessory adaptor that can activate PI3K and may be particularly important with deletion or inhibition of IRS-1 (13). Activation of PI3K leads to phosphorylation of membrane phosphatidylinositol 4,5-bisphosphate $\left[\mathrm{PI}(4,5) \mathrm{P}_{2}\right]$, which generates $\mathrm{PI}(3,4,5) \mathrm{P}_{3}$, some of which is converted to phosphatidylinositol 3,4 -trisphosphate $\left[\mathrm{PI}(3,4) \mathrm{P}_{2}\right]$ by a phospholipid phosphatase. $\mathrm{PI}(3,4) \mathrm{P}_{2}$ and $\mathrm{PI}(3,4,5) \mathrm{P}_{3}$ recruit Akt and phosphoinositide-dependent kinase-1 (PDK1) to the cell membrane by binding their pleckstrin homology domains, which leads to phosphorylation and activation of Akt (14-16). Because PDK1 is constitutively active, movement of Akt to the sarcolemmal membrane is sufficient for activation, and incorporation of the src myristoylation signal creates a constitutively active Akt mutant (8), which then distributes throughout the cell (ref. 17 and data not shown). Of note, although many of the prosurvival benefits of PI3K in the heart have been ascribed to activation of Akt, PI3K also signals through other effectors that are structurally similar to Akt, share overlapping substrate specificity, and promote cell survival in other systems, such as serum- and glucocorticoid-regulated kinase-1 (SGK1) (18). Interestingly, we have 


\section{Table 1}

Baseline cardiac function of ex vivo perfused hearts from myr-Akt, dn-Akt, and NTg mice

\begin{tabular}{lccc}
\hline & $\begin{array}{c}\mathbf{N T g} \\
(\boldsymbol{n}=\mathbf{4 4})\end{array}$ & $\begin{array}{c}\text { myr-Akt } \\
(\boldsymbol{n}=\mathbf{4 4 )}\end{array}$ & $\begin{array}{c}\text { dn-Akt } \\
(\boldsymbol{n}=\mathbf{6})\end{array}$ \\
LVSP, mmHg & $116.7 \pm 2.6$ & $122.1 \pm 3.8$ & $109.1 \pm 6.3$ \\
$\mathrm{LVEDP}, \mathrm{mmHg}$ & $5.2 \pm 0.3$ & $5.7 \pm 0.4$ & $7.3 \pm 0.6^{\mathrm{A}}$ \\
$\mathrm{LVDP}, \mathrm{mmHg}$ & $111.5 \pm 2.7$ & $116.4 \pm 3.9$ & $101.8 \pm 6.6$ \\
$\mathrm{LV} \mathrm{dP/d} t_{\max }, \mathrm{mmHg} / \mathrm{s}$ & $5,593 \pm 220$ & $6,336 \pm 250^{\mathrm{B}}$ & $4,578 \pm 409$ \\
$\mathrm{LV} \mathrm{d} P / \mathrm{d} t_{\min }, \mathrm{mm} \mathrm{Hg} / \mathrm{s}$ & $4,675 \pm 182$ & $6,288 \pm 227 \mathrm{C}$ & $4,535 \pm 453$ \\
Heart rate, bpm & $392 \pm 7$ & $372 \pm 8$ & $358 \pm 8$ \\
$\mathrm{CF}, \mathrm{ml} / \mathrm{min}$ & $3.1 \pm 0.2$ & $4.0 \pm 0.2^{\mathrm{D}}$ & $3.5 \pm 0.6$ \\
\hline
\end{tabular}

$\mathrm{LV} \mathrm{d} P / \mathrm{d} t_{\max }$ and $\mathrm{d} P / \mathrm{d} t_{\min }$, maximum and minimum first derivatives of the LV pressure; CF, coronary flow. ${ }^{A} P<0.05$, dn-Akt versus NTg. $B P<0.05$ and $C P<0.01$, myr-Akt versus dn-Akt and NTg. $D P<0.01$, myr-Akt versus NTg.

recently found that SGK1 is dynamically regulated in the heart and promotes cardiomyocyte survival (19).

To better understand the effects of chronic Akt activation in the heart, we generated $\mathrm{Tg}$ mice (20) with cardiac-specific expression of the same myristoylated Akt (myr-Akt) construct that protects cardiomyocytes in vitro (8) and in vivo (10). These mice have concentric cardiac hypertrophy without overt signs of cardiac disease (20). In the current study, we examined the response of hearts from these mice in an ex vivo model of IRI. We found that chronic Akt activation resulted in decreased functional recovery and increased injury after IRI. The mechanisms and implications of this observation were explored in further detail.

\section{Results}

Baseline cardiac function. Baseline function in hearts from Tg myrAkt (myr-Akt-Tg) and dominant-negative Akt (dn-Akt-Tg) and nontransgenic (NTg) mice was examined in the ex vivo Langendorff model (Table 1). myr-Akt-Tg hearts manifested moderate increases in both positive and negative $\mathrm{d} P / \mathrm{d} t$. Coronary flow was also increased in myr-Akt-Tg mice. The mean LV systolic pressure (LVSP) and LV end-diastolic pressure (LVEDP), LV developed pressure (LVDP), and heart rate were not significantly different compared with those in NTg mice. dn-Akt-Tg hearts manifested modestly increased LVEDP but otherwise comparable cardiac function at baseline. Because echocardiography had previously suggested a nonsignificant trend toward decreased cardiac function in myrAkt-Tg mice in vivo (20), we performed invasive hemodynamic analyses of myr-Akt-Tg compared with NTg mice (Table 2). Isovolumic contraction (e.g., maximum $\mathrm{d} P / \mathrm{d} t$ ) was similar between groups. However, ejection-phase parameters such as end-systolic elastance were impaired in myr-Akt-Tg hearts, which contributed to reduced stroke work and power output. These data are consistent with a moderate reduction in cardiac function in vivo. Differences from the ex vivo results likely reflect differences in afterload, neurohumoral input, and anesthetic effects.

Functional recovery after IRI. After 20-minute control perfusion, hearts were exposed to 20-minute global ischemia followed by 40-minute reperfusion, while cardiac function was monitored in the Langendorff model (Figure 1, A and B). In NTg hearts, LVDP recovered to $53.5 \% \pm 3.4 \%$ of its starting value during reperfusion. dn-Akt-Tg hearts manifested moderately impaired recovery, achieving a maximum LVDP $34.4 \% \pm 7.5 \%$ of baseline, consistent with a cardioprotective role for endogenous Akt. In contrast, no cardiac function was restored in any of the myrAkt-Tg hearts, a result that was significantly worse than those in either the NTg or dn-Akt hearts.

Cardiac injury after IRI. The activity of creatine phosphokinase $(\mathrm{CPK})$ released into the perfusate during reperfusion was measured as an index of injury. During control perfusion, no CPK activity was detectable (data not shown). After IRI, significant CPK activity was detectable in the perfusate of NTg hearts, and there was a nonsignificant trend toward increased CPK in dnAkt-Tg hearts ( $P=0.068$ vs. NTg; Figure $1 \mathrm{C})$. Remarkably, CPK was increased 35-fold in myr-Akt-Tg compared with NTg hearts $(P<0.005$; Figure 1C). This correlated with dramatically larger infarcts in myr-Akt-Tg hearts $(80.5 \% \pm 3.1 \%$ vs. $32.6 \% \pm 9.8 \%$; $P<0.005$; Figure 1D). After IRI, there was a nonsignificant trend toward reduced DNA laddering in myr-Akt-Tg hearts at both 5 and 40 minutes of reperfusion (Figure 1E). Thus, the increased injury seen in myr-Akt-Tg hearts is not due to increased apoptosis but may reflect an increase in other forms of cell death.

IRS-1/PI3K signaling is markedly inbibited in myr-Akt-Tg mice. To explore possible mechanisms for these results, we examined signaling in myr-Akt-Tg compared with NTg hearts. As noted previously, no difference was seen in MAPK pathways (20). While baseline activities of immunoprecipitated PI3K isoforms $(110-\alpha,-\beta,-\gamma)$ were comparable (Supplemental Figure 1; supplemental material available online with this article; doi:10.1172/JCI23073DS1), IRS-1associated PI3K activity was dramatically inhibited both at baseline and after either insulin stimulation or ischemia/reperfusion (Figure 2, A and B; Supplemental Figure 2). This reduced activity correlated with reduced IRS-1-associated PI3K in myr-Akt-Tg hearts even after insulin stimulation (Figure 2C).

While PI3K expression was comparable in myr-Akt-Tg and NTg mice, IRS-1 was reduced in myr-Akt-Tg mice (Figure 2D), which suggests that loss of IRS-1 largely accounts for decreased IRS-1-associated PI3K activation. We also examined IRS- 1 in an independent, X-linked (TG20) line of myr-Akt-Tg mice with lower levels of Akt expression and activation (20). Male TG20 mice also exhibited a significant though less-marked reduction in IRS-1, which was further attenuated in chimeric hearts of female TG20 mice, consistent with an Akt dosage effect (Figure 2D).

Serine phosphorylation of IRS-1 can both inhibit PI3K activation (21) and induce IRS-1 degradation (22). Using an antibody

\section{Table 2}

Baseline cardiac function based on in vivo hemodynamic analysis

$\begin{array}{lcc} & \mathbf{N T g}(\boldsymbol{n}=\mathbf{3}) & \text { myr-Akt }(\boldsymbol{n}=\mathbf{3}) \\ \text { LVSP, } \mathrm{mmHg} & 88.3 \pm 3.9 & 95.5 \pm 9.2 \\ \mathrm{LVEDP}, \mathrm{mmHg} & 5.6 \pm 1.6 & 10.5 \pm 2.0 \\ \mathrm{LVDP}, \mathrm{mmHg} & 82.7 \pm 4.3 & 85.1 \pm 10.0 \\ \mathrm{LV} \mathrm{d} P / \mathrm{d} t_{\max }, \mathrm{mmHg} / \mathrm{s} & 9,472 \pm 751 & 7,321 \pm 1,091 \\ \mathrm{LV} \mathrm{d} P / \mathrm{d} t_{\min }, \mathrm{mm} \mathrm{Hg} / \mathrm{s} & 8,903 \pm 355 & 6,209 \pm 1,222 \\ \text { Heart rate, bpm } & 481 \pm 13 & 507 \pm 28 \\ \text { Ees/HW, } \mathrm{mmHg} / \mu \mathrm{l} / \mathrm{g} & 40.3 \pm 2.6 & 20.5 \pm 6.0^{\mathrm{A}} \\ \mathrm{SW}, \mathrm{mmHg} \times \mu \mathrm{l} & 2,024 \pm 280 & 746 \pm 242^{\mathrm{B}} \\ \text { PwrMaxEDV, mmHg/s } & 25.8 \pm 3.8 & 13.2 \pm 2.1^{\mathrm{A}}\end{array}$

Ees/HW, end-systolic elastance normalized by heart weight; SW, stroke work; PwrMaxEDV, maximal ventricular power normalized to end-diastolic volume. ${ }^{A} P<0.05$ and ${ }^{B} P<0.02$ versus $N T g$. 
A

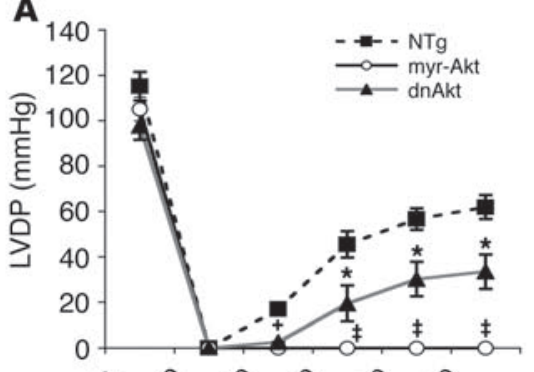

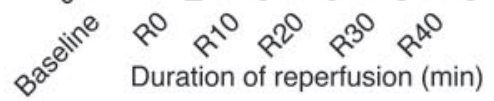

\section{D}
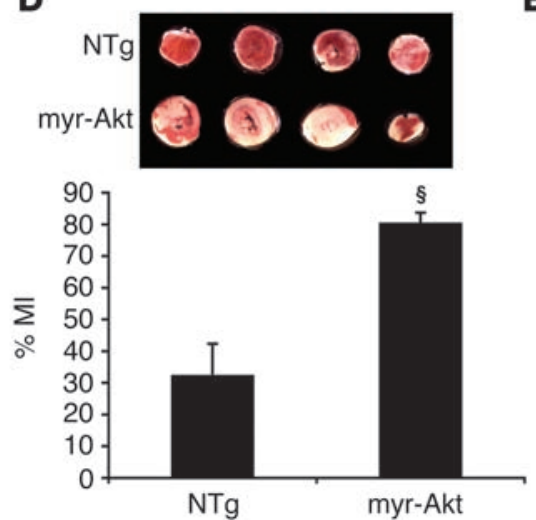

B

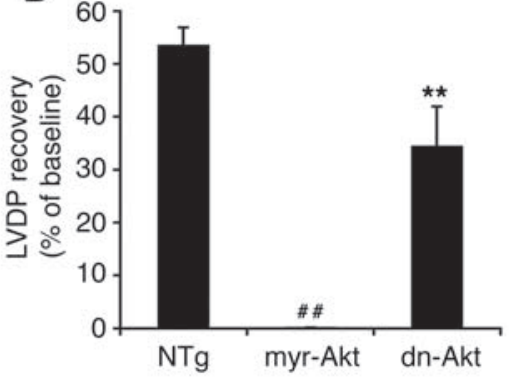

C

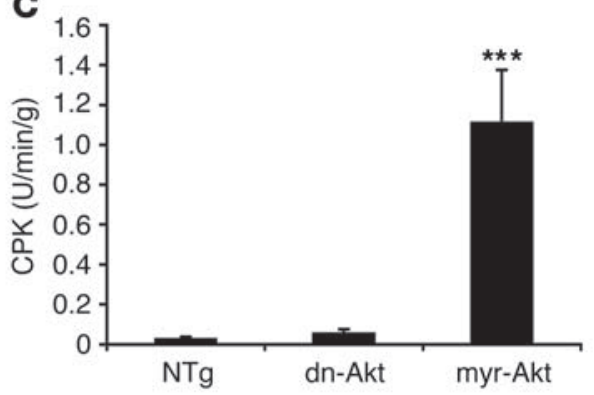

E NTg myr-Akt

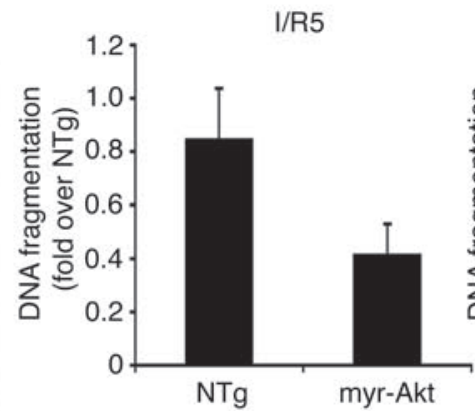

I/R40

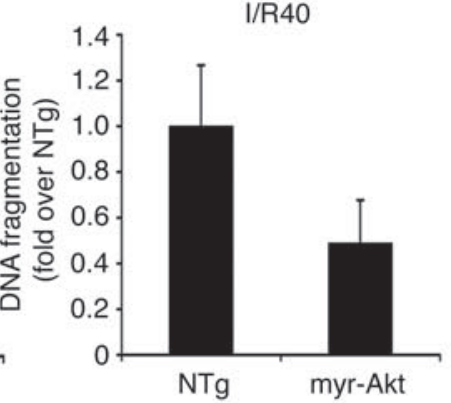

I/R40

Figure 1

Chronic Akt activation in myr-Akt-Tg mice has detrimental effects on cardiac functional recovery and injury after ischemia/reperfusion. (A) LVDP profiles during ischemia/reperfusion in NTg (filled squares; $n=11$ ), myr-Akt (open circles; $n=11$ ), and dn-Akt mice (filled triangles; $n=5$ ). ${ }^{\dagger} P<0.01$ and $\ddagger P<0.001$, myr-Akt versus NTg; ${ }^{*} P<0.001$, dn-Akt versus NTg. (B) Maximum LVDP recovery (percent of baseline) measured at 40 minutes of reperfusion. ${ }^{\# \#} P<0.0001$ versus NTg; ${ }^{* \star} P<0.02$ versus NTg. (C) CPK values in the effluent collected during reperfusion period (NTg, $n=6$; dn-Akt, $n=4$; myr-Akt, $n=7$ ). ${ }^{* *} P<0.02$ versus dn-Akt. (D) Micrograph showing representative TTC staining of cardiac sections from NTg (top row) and myr-Akt (bottom row) mice. Effects on quantitated cumulative infarct area size in myr-Akt compared with NTg mice $(n=3$ each) are represented in the bar graph. $\$ P<0.005$ versus NTg. \%MI, myocardial infarct area/ventricular area. (E) Representative DNA laddering indicating the degree of apoptosis in perfused heart tissues from myr-Akt and NTg mice $(n=3$ each) after 20 minutes ischemia/40 minutes reperfusion (I/R40). Bar graphs depict quantitation of DNA ladder density and represent results of 3 independent experiments after 20 minutes of ischemia and either 5 (I/R5) or 40 minutes of reperfusion.

specific for the phosphorylated form of the Akt consensus substrate (catalog 9611; Cell Signaling Technology), we saw a relative increase (compared with the reduction in total IRS-1) in Akt target phosphorylation migrating at the same position as immunoreactive IRS-1 in myr-Akt-Tg hearts (Figure 2E). These observations raise the possibility that Akt phosphorylation of IRS-1 could contribute to IRS-1 degradation. To see whether these effects simply reflect the nonphysiological activation of Akt in our Tg mice, we examined IRS-1 in hearts from Akt1-null mice (23). While impaired placental development and fetal growth in these mice limited the numbers available for study, immunoblotting revealed an increase in IRS-1 protein levels that was substantial in $\mathrm{Akt}^{-/-}$and more modest in $\mathrm{Akt} 1^{+/-}$hearts (Figure 2F). IRS-1 levels in dn-Akt-Tg were not significantly different from those in NTg hearts. This difference may reflect the incomplete inhibition of Akt 1 signaling seen in these mice (Supplemental Figure 3) that only comes on in the ventricle after birth. In contrast, Akt1/- mice have complete loss of Akt 1 signaling, and $\mathrm{Akt} 1^{+/-}$mice exhibit moderate reduction in Akt 1 that is present throughout development. Interestingly, IRS-1 mRNA was also reduced by $68.7 \%$ in myr-Akt-Tg $(n=7)$ compared with NTg hearts $(n=5 ; P<0.001)$.
Thus, loss of IRS-1 in myr-Akt-Tg correlates with Akt activation and reflects physiological IRS-1 regulatory mechanisms that are enhanced in the myr-Akt-Tg and lost in the Akt-null mice.

IRS- 2 can also activate PI3K and may be particularly important in the setting of reduced IRS-1 levels (13). Interestingly, IRS-2 mRNA and protein levels were also reduced in myr-Akt-Tg according to quantitative RT-PCR (QRT-PCR) and immunoblotting: $59.9 \%$ and $42.4 \%$ respectively $(P<0.005$ and $P<0.02$, respectively). Thus, in addition to reducing the primary adaptor activating the isoform of PI3K including the $110 \alpha$ catalytic domain (PI3K $\alpha$ ), Akt1 activation leads to inhibition of the accessory pathway, thereby severely impairing PI3K activation.

Akt activation induces proteasome-dependent degradation of IRS-1. To further explore the mechanism of reduced IRS-1 protein, we examined neonatal rat cardiomyocytes (NRCMs) in vitro (Figure 3). Stimulation with either insulin or IGF-1 (data not shown) for 20 hours triggered a reduction in IRS- 1 protein levels, as did stimulation with adenoviral myr-Akt (Ad.myr-Akt; Figure 3A). In both cases, IRS-1 degradation was inhibited by the proteasome inhibitor ALLN but not the structural control, ALLM, a protease inhibitor with poor affinity for the proteasome (24). To examine 
A IP: IRS-1
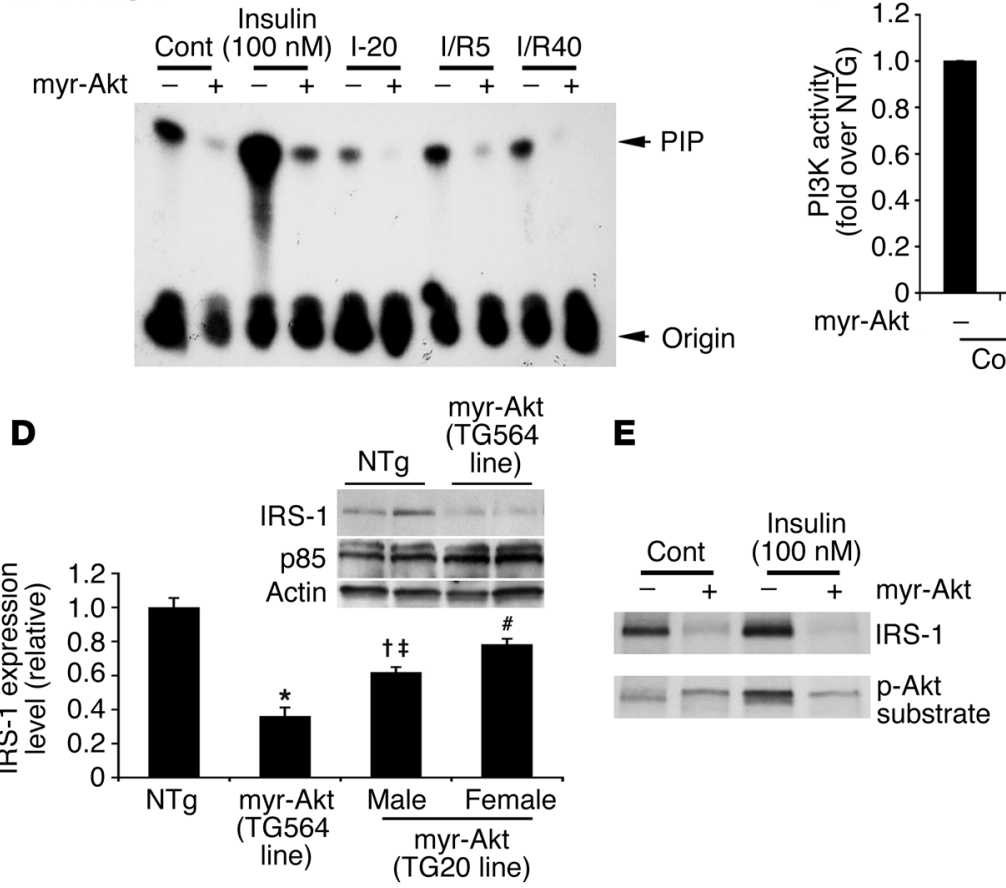

B

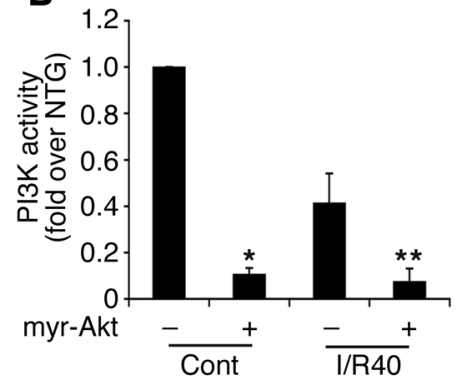

C

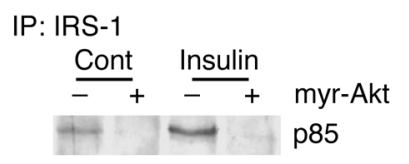

$\mathbf{F}$

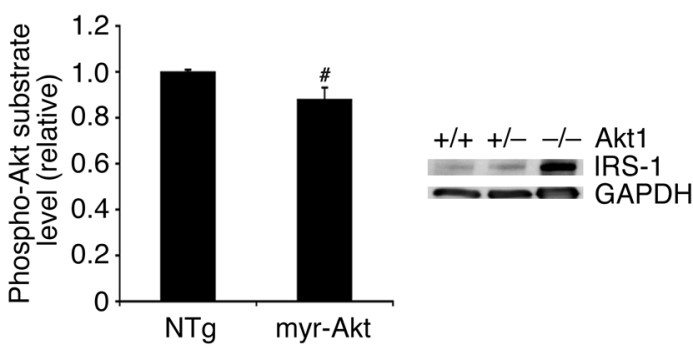

Figure 2

IRS-1/PI3K signaling is inhibited in myr-Akt hearts. (A) IRS-1-associated PI3K activity was analyzed in the hearts perfused without (Cont, $n=5$ ) or with insulin ( $100 \mathrm{nM}, 10$ minutes; $n=3$ ) or subjected to 20 minutes of ischemia (I-20) followed by 5 minutes or 40 minutes of reperfusion. PIP, phosphoinositide; origin, initial position of the reaction mixer. (B) Quantitation of mean spot density from cumulative data are shown for control ( $n=5$ each) and ischemia/reperfusion ( $n=3$ each) normalized to the values for the control-perfused NTg hearts. ${ }^{*} P<0.001$ versus control-perfused NTg; ${ }^{*} P<0.01$ versus NTg after ischemia/reperfusion. (C) Lysates from the hearts with or without insulin stimulation were immunoprecipitated with IRS-1 and immunoblotted for the p85 subunit of PI3K. Representative data from 4 independent experiments are shown. (D) Representative immunoblot for IRS-1 and p85 from cardiac lysates of myr-Akt (TG564 line) $(n=6)$ or NTg $(n=5)$ mice. Actin was included as a loading control. Densitometric quantitation from the different line of myr-Akt-Tg (TG20 line, male and female; $n=3$ each) in addition to TG564 line is shown in the bar graph. ${ }^{*} P<0.001$ versus NTg; ${ }^{\dagger} P<0.01$ versus NTg and TG564; $¥ P<0.02$ versus TG20 female; $\# P<0.05$ versus NTg. (E) Lysates from the hearts with or without insulin stimulation were immunoblotted first with anti-IRS-1 antibody and subsequently with anti-phosphoAkt substrate antibody. Representative data from 4 independent experiments are shown. Densitometric quantitation from control hearts is shown in the bar graph. (F) Representative immunoblots of IRS-1 from cardiac lysates of Akt1 ${ }^{+/+}$, Akt $1^{+/-}$, and Akt $1^{-/-}$mice are shown from 3 independent experiments. GAPDH immunoblotting is shown as a loading control.

IRS-1 phosphorylation, we infected NRCMs with Ad.IRS-1 and the indicated adenoviral vectors. Immunoprecipitation of IRS-1 followed by immunoblotting for phosphorylated Akt substrate revealed an increase in phosphorylated IRS-1 levels after insulin stimulation or expression of myr-Akt but not dn-Akt or control virus (Figure 3B). Thus, Akt activation is sufficient to induce IRS-1 phosphorylation and proteasome-dependent degradation in cardiomyocytes.

IRS-1 is preserved in IGF-1-Tg mice. Given the results described above, one might anticipate that IRS- 1 would also be reduced in IGF-1-Tg mice. Since IGF-1-Tg mice are protected against injury in IRI (7), a similar reduction in IRS-1 and/or IRS-1-associated PI3K would argue against a pathophysiological role for these signaling changes. Importantly, IGF-1-Tg mice demonstrate not only reduced apoptosis after IRI (7) but also improved functional recovery compared with NTg mice (LVDP, $72.6 \% \pm 4.4 \%$ of baseline vs. $52.3 \% \pm 2.3 \% ; P<0.002$; Figure $4 \mathrm{~A}$ ). Interestingly, IRS-1-associated PI3K activity was increased in IGF-1-Tg hearts at baseline and showed a nonsignificant trend toward higher activity after ischemia/reperfusion (Figure 4B). IRS-1 protein levels were comparable in IGF-1-Tg and NTg mice (Figure 4C).
Similarly, gene transfer of IGF-1 in NRCMs did not induce the IRS-1 degradation evident with acute IGF-1 treatment (data not shown). Thus, while Akt activation induces degradation of IRS-1 and loss of Akt 1 increases cardiac IRS-1, tonic expression of IGF-1 both in vitro and in vivo appears to escape this regulation, leaving open the possibility that loss of PI3K contributes to increased injury in myr-Akt-Tg mice.

In vivo cardiac gene transfer of activated PI3K rescues function and reduces injury in myr-Akt-Tg hearts after IRI. To test the functional significance of reduced IRS-1-associated PI3K activity, we performed in vivo cardiac gene transfer with Ad.BD110 (8), encoding constitutively active PI3K $\alpha$ (25), the isoform linked to IRS signaling. Three days after gene transfer, BD110 expression was evident by immunoblotting for the incorporated $c$-myc epitope in Ad.BD110-infected hearts but not in hearts infected with control virus (Figure 5A). Importantly, Ad.BD110 restored PI3K activity in myr-Akt-Tg mice to a level comparable to that in control mice after IRI (Figure 5B). After gene transfer, hearts were subjected to 30-minute global ischemia and 40-minute reperfusion. As before, no functional recovery was seen in uninfected or control virus-infected myr-Akt-Tg mice (Figure 5, C and D). 


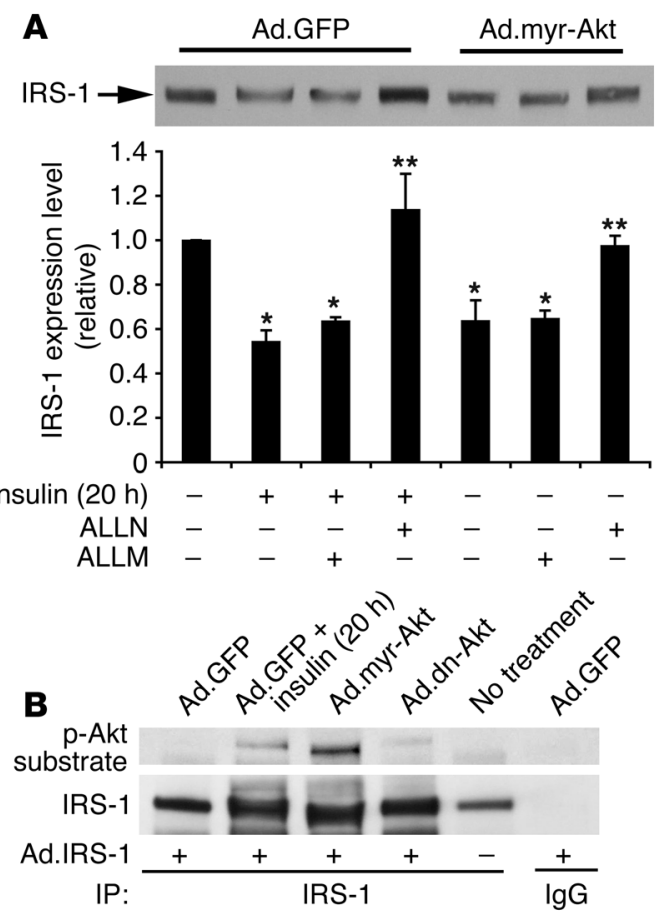

In contrast, after Ad.BD110 infection, myr-Akt-Tg mice demonstrated substantial functional recovery, achieving a maximal LVDP of $34.1 \% \pm 2.0 \%$, significantly better than that of uninfected myr-Akt-Tg but not quite as good as that of NTg mice (maximal LVDP, $41.8 \% \pm 1.9 \%$; Figure 5, C and D). Remarkably, tissue

\section{Figure 3}

Insulin- or myr-Akt-induced proteasomal degradation of IRS-1. (A) NRCMs were treated as described in Methods. A representative immunoblot for IRS-1 and densitometric quantitation normalized to control virus-infected, untreated cells are shown. IRS-1 degradation induced either by insulin stimulation $(n=6)$ or Ad.myr-Akt infection $(n=3)$ was inhibited by ALLN. ${ }^{*} P<0.001$ versus untreated control cells; ${ }^{* \star} P<0.001$ ALLM-treated versus ALLN-treated NRCMs stimulated in parallel. (B) NRCMs were cotransfected with Ad.IRS-1 and the indicated adenoviruses. Insulin (100 nM) was added as indicated 20 hours before harvest. Total cell lysates were immunoprecipitated with anti-IRS-1 antibody and immunoblotted first with anti-phosphoAkt substrate antibody and subsequently with anti-IRS-1 antibody. No immunoreactive bands for IRS-1 or phospho-Akt substrate were seen after immunoprecipitation with control lgG. injury as indicated by CPK release was also dramatically reduced by Ad.BD110 gene transfer (Figure 5E). IRS-1 expression was not affected by Ad.BD110, which suggests that restoration of PI3K activity is responsible for the observed rescue despite persistently reduced IRS-1 levels (Figure 5F). MAPK signaling was not altered by Ad.BD110 (data not shown). Akt activity was increased in myr-Akt-Tg compared with NTg mice but did not increase further after Ad.BD110 gene transfer, which likely reflects saturation of this signaling pathway in myr-Akt-Tg mice (Figure 5G). Interestingly, BD110 did increase phosphorylation (inhibition) of endogenous glycogen synthase kinase- $3 \beta$ (GSK3 $\beta$ ) (Figure 5G). Although baseline GSK3 $\beta$ phosphorylation

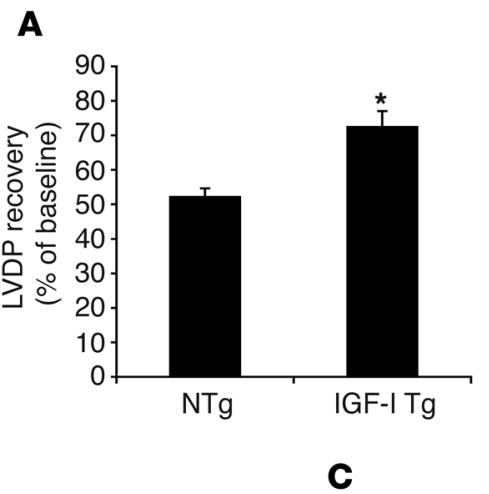

IRS-1 IGF-I NTg
B IP: IRS-1
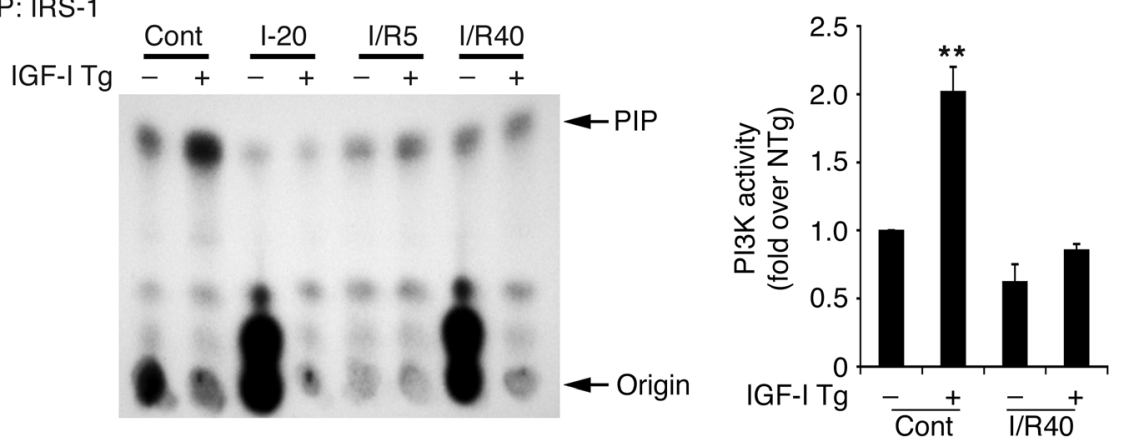

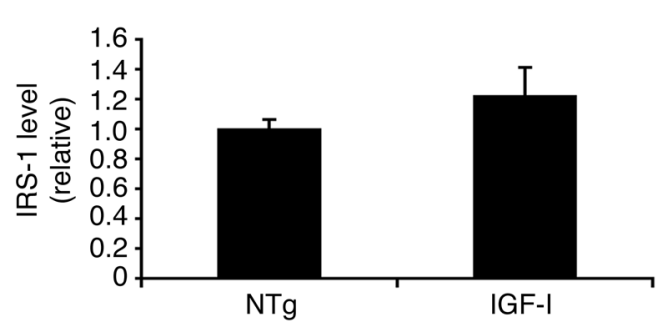

Figure 4

IGF-1-Tg mouse hearts show improved functional recovery after ischemia/reperfusion with enhanced activation of PI3K. (A) Maximum LVDP recovery relative to baseline was measured at 40 minutes of reperfusion after 20 minutes of ischemia. ${ }^{\star} P<0.002$ versus NTg; $n=6$ each. (B) IRS-1-associated PI3K activity in IGF-1-Tg and NTg hearts. Left: A representative experiment is shown. Right: Cumulative data from 4 independent experiments are expressed relative to data for control-perfused NTg hearts. ${ }^{* \star} P<0.001$ versus control-perfused NTg. (C) Representative immunoblot for IRS-1 in cardiac lysates of IGF-1-Tg and NTg mice is shown (left). In the bar graph, quantitation relative to the value of NTg of 3 independent experiments is shown. 

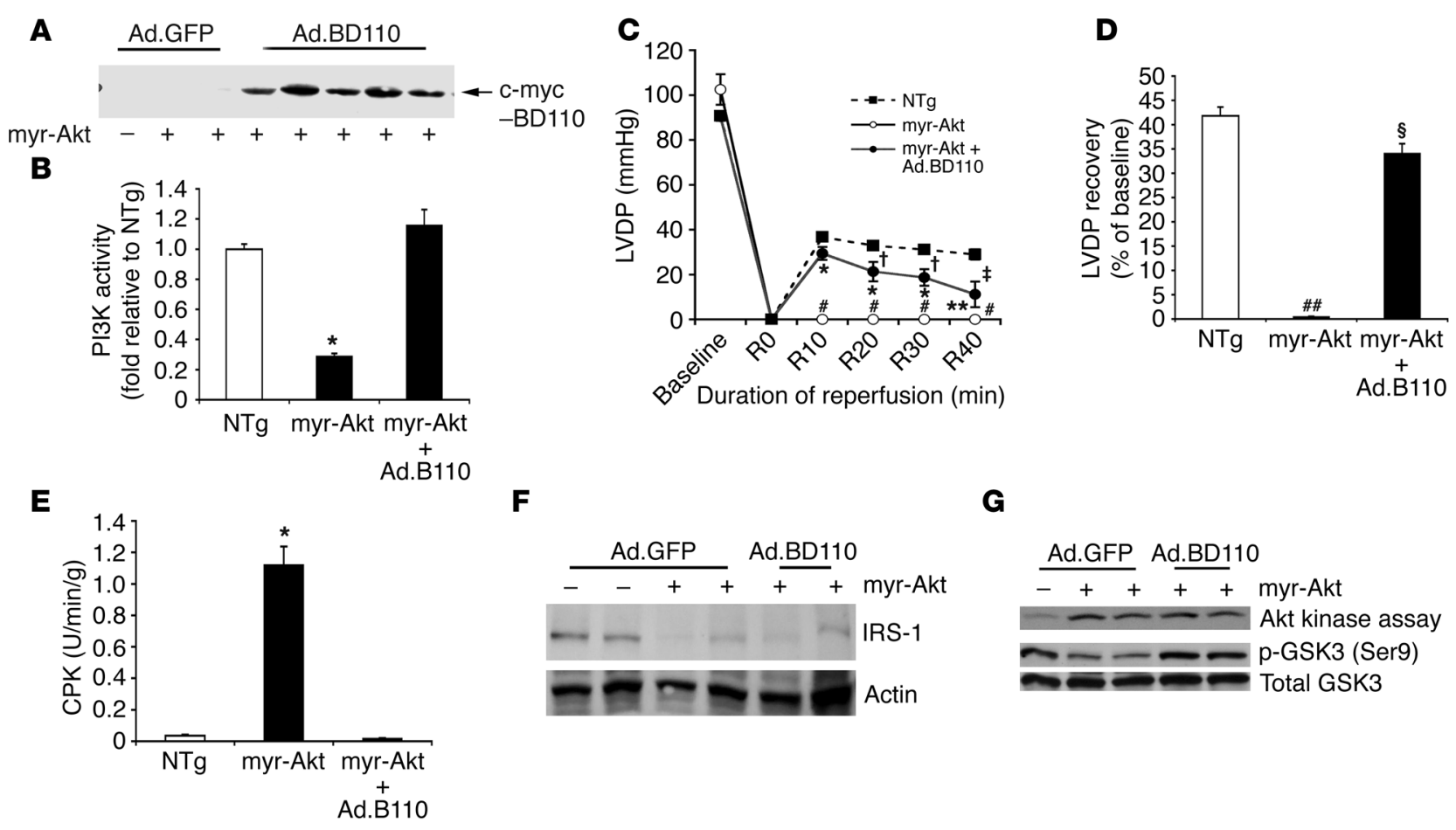

G

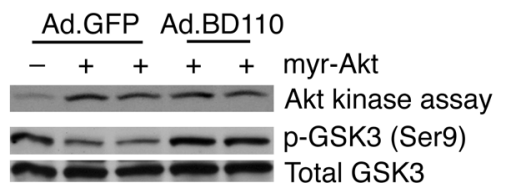

\section{Figure 5}

Ad.BD110 rescues cardiac function and reduces injury after ischemia/reperfusion. (A) Immunoblotting reveals the c-myc epitope incorporated into BD110 only in Ad.BD110-injected myr-Akt-Tg hearts. (B) After immunoprecipitation with anti-p110 $\alpha$ antibody, PI3K activity was measured in immune complexes using the fluorescence polarization assay. Results are expressed as a ratio to the mean value for NTg hearts (NTg, $n=10$; myr-Akt alone, $n=5$; myr-Akt with Ad.BD110, $n=6$ ). ${ }^{*} P<0.001$ versus NTg or Ad.BD110-injected myr-Akt hearts. (C) LVDP profiles during ischemia/reperfusion in NTg (filled squares; $n=13$ ), myr-Akt (open circles; $n=8$ ), and myr-Akt with Ad.BD110 injection (filled circles; $n=7$ ). ${ }^{\sharp} P<0.001$ versus NTg; ${ }^{*} P<0.001$ and ${ }^{* *} P<0.05$ versus myr-Akt alone; ${ }^{\dagger} P<0.01$ and $\neq P<0.001$ versus NTg. (D) Maximum LVDP recovery achieved (percent of baseline) after ischemia/reperfusion. \#\# $P<0.0001$ versus NTg or Ad.BD110-injected myr-Akt; $\$ P<0.02$ versus NTg. (E) CPK activity in the effluent (NTg, $n=7$; myr-Akt, $n=5$; myr-Akt with Ad.BD110, $n=4)$. (F) Cardiac lysates were immunoblotted for IRS-1 expression. Actin was included as a loading control. (G) Cardiac lysates were analyzed for Akt kinase activity with GSK3 $\alpha / \beta$ as substrate. Lysates from the same heart tissues were subjected to immunoblotting of phospho- and total GSK3.

in myr-Akt-Tg hearts is comparable to that in NTg hearts (20), the increase in GSK3 $\beta$ phosphorylation normally seen after IRI was not observed in myr-Akt-Tg hearts, which reflects the inhibition of IRS/PI3K signaling (Figure 5G). After restoration of PI3K activity, GSK3 $\beta$ phosphorylation is increased in the absence of further enhanced Akt activation. Thus, GSK3 $\beta$ phosphorylation occurs in IRI via IRS/PI3K-dependent signaling, and BD110 restores GSK3 $\beta$ phosphorylation through PI3K-dependent but Akt-independent pathways. Of note, GSK3 $\beta$ inhibition with $\mathrm{LiCl}$ and the specific inhibitor SB216763 did not restore function to myr-Akt-Tg hearts during IRI (Supplemental Figure 4). Thus, acute GSK3 $\beta$ inhibition is not sufficient to restore cardioprotection, which possibly reflects the involvement of other pathways or a requirement for longer-term inhibition.

SGK1 phosphorylation is inhibited in myr-Akt-Tg hearts. SGK1 is phosphorylated and activated by PI3K and promotes cardiomyocyte survival in vitro (19). Phosphorylation of SGK1 was decreased in myr-Akt-Tg hearts (Figure 6), which confirms impaired Akt-independent PI3K signaling.

IRS-1 expression is reduced in failing buman hearts. To investigate whether a similar loss of IRS-1 occurs in human heart failure, we examined samples from nondiabetic, failing human hearts or unused donor hearts as controls. As previously reported (11), Akt activity was increased in heart failure samples (Figure 7A). Intriguingly, IRS-1 protein was significantly decreased in failing hearts compared with controls, with relative preservation of comigrating phospho-Akt-substrate (Figure 7B).

\section{Discussion}

Here we show that chronic Akt activation has detrimental effects after IRI due to feedback inhibition of IRS/PI3K signaling. This mechanistic connection is demonstrated by the rescue of function and reduced injury seen after restoration of PI3K signaling. Moreover, while the primary defect appears to be a reduction in IRS protein levels, these rescue experiments suggest the adverse consequences observed in the heart arise from the inability to activate PI3K. These data also demonstrate that PI3K-dependent but Akt-independent pathways are crucial for full cardioprotection and suggest a mechanism by which chronic Akt activation may become maladaptive.

Although our myr-Akt-Tg mice undoubtedly represent a particularly dramatic example of Akt-driven feedback inhibition of IRS/PI3K signaling, several observations suggest that these findings have broader physiological relevance. First, IRS-1 levels were increased in Akt1-null mice, which suggests that Akt is a physiological regulator of IRS-1. Second, activation of endogenous 


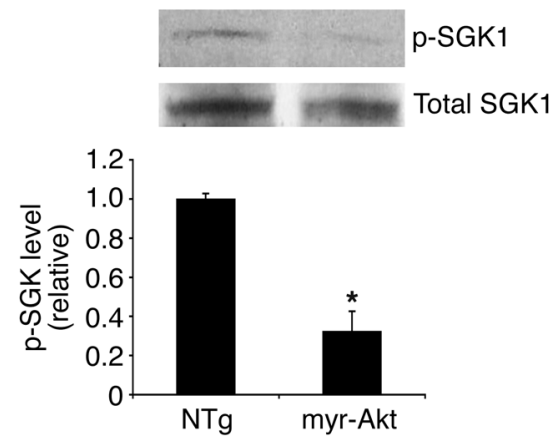

Figure 6

Phosphorylation of SGK1 is reduced in myr-Akt hearts. Representative immunoblot for phospho- and total SGK1 from cardiac lysates of myrAkt-Tg or NTg mice ( $n=3$ each). Densitometric quantitation is shown in the lower panel. ${ }^{*} P<0.002$ versus NTg.

Akt in cardiomyocytes by stimulation with insulin or IGF-1 also led to a decrease in IRS-1 in vitro. Finally, cardiac samples from patients with heart failure in which Akt is activated (11) also demonstrated a reduction in IRS-1. Moreover, these data are consistent with evidence that cardiac IRS- 1 is decreased in type 2 diabetes $(26,27)$, which possibly represents a response to chronic hyperinsulinemia and contributes to the development of insulin resistance in this setting. Prior studies in other systems have also demonstrated that serine or threonine phosphorylation of IRS- 1 mediated by a variety of kinases including inhibitor of $\kappa \mathrm{B}$ kinase $(\mathrm{IKK})(28,29)$, PKC $(30,31)$, JNK (32), p70S6 kinase (33), and Akt (21) can inhibit PI3K activation through either reduced IRS-1/PI3K association or degradation. The current study extends these observations by demonstrating that Akt-driven feedback inhibition of IRS/PI3K signaling occurs in cardiomyocytes and that it has dramatic adverse consequences during IRI. We also found that mRNA levels of IRS-1 and IRS-2 were reduced in myr-Akt-Tg hearts, which is consistent with prior reports indicating that insulin signaling can inhibit IRS-2 transcription through an insulin response element (IRE) $(34,35)$. A similar IRE exists in the IRS1 gene (36), although its functional role has not been demonstrated. In IRS-1-null mice, IRS-2 functions as an accessory adaptor molecule mediating activation of PI3K (13). In contrast, IRS-2 is also reduced with chronic Akt activation, which thereby precludes this potential compensatory mechanism. Thus, feedback inhibition of IRS/PI3K signaling occurs through multiple mechanisms and likely reflects a physiological regulatory role of Akt. These findings may have relevance for a variety of conditions in which Akt is chronically activated in the heart, including heart failure. In addition, these studies have implications for genetic or other therapies targeted at Akt activation and suggest that long-term activation of this pathway would eventually have adverse consequences.

The mammalian target of rapamycin (mTOR) and its target, p70S6 kinase, represent an important effector pathway downstream of IRS/PI3K and are activated in myr-Akt-Tg mice (20). Moreover, mTOR activation of p70S6 kinase can mediate feedback inhibition of IRS/PI3K signaling (33). Of note, while the PI3K inhibitor wortmannin completely prevented insulininduced loss of IRS-1 in cardiomyocytes, rapamycin only modestly inhibited IRS-1 loss (Supplemental Figure 5). Moreover, rapamycin did not restore functional recovery to myr-Akt-Tg hearts $(n=4$; data not shown). Together these data suggest that $\mathrm{PI} 3 \mathrm{~K} / \mathrm{Akt}$ mediates the effects we observe largely independently of mTOR/p70S6 kinase. Nevertheless, in the current study, we have not identified the IRS-1 phosphorylation site nor demonstrated a direct physical interaction between IRS-1 and Akt, and thus Akt may modulate IRS-1 indirectly.

How do we reconcile these data with numerous studies documenting the benefits of acute Akt activation? We propose the following model. Since the degree of feedback inhibition observed acutely is relatively modest, acute Akt activation confers a net cardioprotective benefit. However, with chronic (or perhaps repetitive) Akt activation, the feedback inhibition of IRS/PI3K becomes more severe. The dramatically increased injury seen in myr-Akt-Tg mice after IRI demonstrates that Akt activation alone is not sufficient for cardioprotection in the face of PI3K inhibition. PI3Kmediated rescue demonstrates that the hypertrophy seen in myrAkt-Tg does not account for the increased injury and supports the importance of PI3K-dependent but Akt-independent cardioprotective pathways (Figure 8). Thus we hypothesize that multiple pathways (Akt dependent and independent) are each necessary for full cardioprotection. Consistent with this model, dn-Akt and Akt1-null hearts exhibited impaired functional recovery after IRI (maximum LVDP, $16.5 \%$ and $14.2 \%$ in $\mathrm{Akt}^{+/-}$and $\mathrm{Akt}^{-/}$, respectively; $n=2$ each). Our studies suggest that the increase in IRS-1 levels seen in these mice (modest in $\mathrm{Akt}^{+/}$and more marked in $\mathrm{Akt}^{-/}$) was offset by the loss of cardioprotective Akt1 signaling. We hypothesize that as additional pathways are identified, inhibition of each would compromise recovery in IRI but no single pathway would be sufficient for full cardioprotection.

Interestingly, the X-linked TG20 line has demonstrated acute cardioprotection in vivo (20). This line, which expresses less of

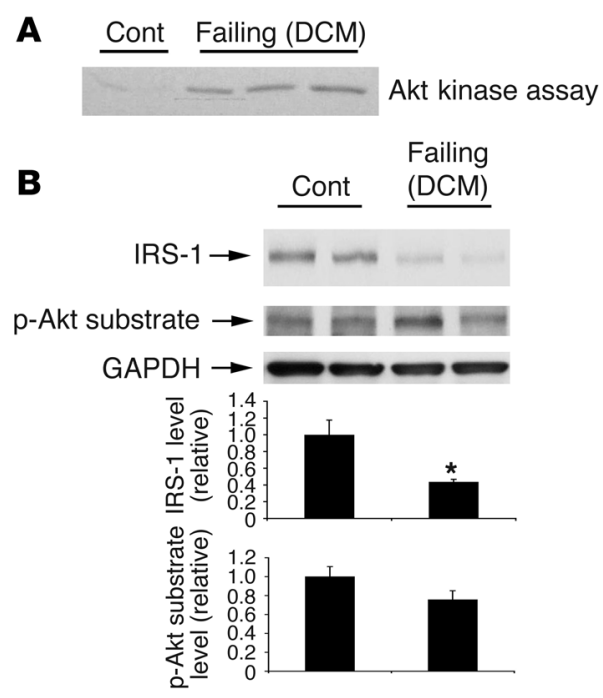

Figure 7

IRS-1 protein is reduced in failing human hearts. (A) Total tissue lysates from indicated samples were analyzed for Akt kinase activity with GSK $\alpha / \beta$ as substrate. (B) Total tissue lysates from either failing hearts $(n=5)$ or control donor hearts $(n=4)$ were immunoblotted with anti-IRS-1 antibody and subsequently with anti-phospho-Akt substrate antibody. In the lower panel, densitometric quantitations for IRS- 1 level and $p$-Akt substrate level are shown. ${ }^{*} P<0.005$ versus control. DCM, dilated cardiomyopathy. 


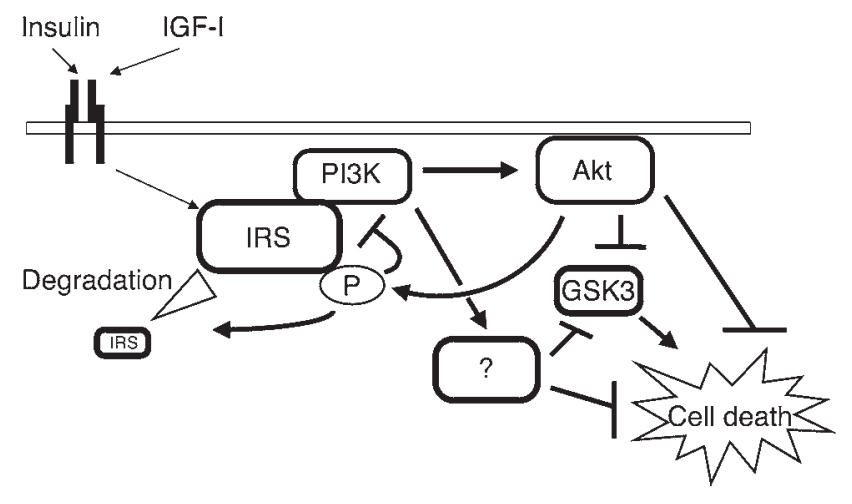

Figure 8

Schematic representation of Akt-mediated feedback inhibition. Aktinduced phosphorylation of IRS proteins may lead to their dissociation from PI3K as well as proteasome-dependent degradation. Simultaneously, IRS transcription is inhibited (data not shown), which further reduces IRS levels. Consequently, activation of PI3K is impaired. Loss of signaling via PI3K-dependent but Akt-independent survival pathways can lead to increased injury despite persistent Akt activation.

the myr-Akt transgene, exhibits a significant but less marked reduction in the level of IRS-1, which is further attenuated in chimeric hearts of female mice (Figure 2D). Thus, we would expect TG20 mice to manifest fewer adverse consequences of chronic Akt activation. In addition, as previously reported, transcript profiling revealed dramatic upregulation (675-fold by QRTPCR) of mRNA for OTT in TG20 but not TG564 hearts (37). OTT is an X-chromosome-encoded gene previously described only in ovary and testis that is likely upregulated by a positional effect of transgene insertion (37). While it is impossible to fully predict the biological effects of this artifact, it has prompted us to focus current efforts primarily on the TG564 line. Finally, a variety of important differences between the in vivo and the ex vivo ischemia/reperfusion models could also contribute to the different observations. These include the relative prevalence of different forms of cell death, as well as systemic influences from vascular tone, neurohumoral input, and the presence of circulating hematopoietic cells. Of note, inhibition of leukocyte recruitment attenuates IRI in vivo $(38,39)$ but has no bearing ex vivo. Since Akt 1 activates eNOS $(40,41)$, NO-mediated inhibition of leukocyte adhesion may mitigate IRI (42) in vivo despite a moderate reduction in the level of IRS-1 in the TG20 line. To better understand intrinsic mechanisms controlling cardiomyocyte survival, we have focused the current investigation on the isolated heart model.

The observation that BD110 increased GSK3 $\beta$ phosphorylation without an increase in Akt activation raises the possibility that these pathways may also phosphorylate GSK $3 \beta$, previously proposed to act as a central integrator of survival signaling $(43,44)$. For example, SGK1 is a PI3K-dependent serine-threonine kinase that can phosphorylate GSK3 $\beta$ and promote survival in cardiomyocytes (19). Decreased SGK1 phosphorylation in myr-Akt-Tg hearts provides evidence for impaired Akt-independent PI3K signaling but does not establish a functional role for SGK1 in this context. Although, as previously reported, GSK3 $\beta$ inhibition improved functional recovery in WT hearts $(43,45)$, it did not rescue function in myr-Akt-Tg hearts (Supplemental Figure 4). This may reflect involvement of other pathways or a requirement for longer-term inhibition.
The current study is consistent with prior work demonstrating that PI3K plays a cardioprotective role in settings such as preconditioning (46). However, acute inhibition of PI3K with wortmannin did not adversely affect recovery in normal hearts (46). Consistent with this, we found that wortmannin did not

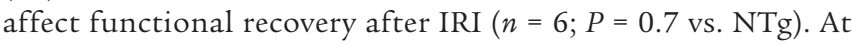
this dose $(100 \mathrm{nM})$, wortmannin completely abrogated Akt phosphorylation but only partially and inconsistently inhibited phosphorylation of GSK3 $\beta$ (Supplemental Figure 6). At a higher dose $(200 \mathrm{nM})$, inhibition of GSK3 $\beta$ phosphorylation was more consistent and functional recovery impaired, but instability in baseline cardiac function precluded quantitative analysis of recovery. Thus, wortmannin may incompletely inhibit the Aktindependent targets of PI3K that continue to mediate GSK3 $\beta$ phosphorylation. Perhaps even more important, PI3K inhibition in myr-Akt-Tg is chronic, and the duration of inhibition likely affects the response to IRI.

Although IRS-1 expression was reduced by insulin or IGF-1 stimulation in vitro, as well as Akt activation in vivo, IRS-1 was preserved and associated with higher baseline PI3K activation in IGF-1-Tg mice. The protection seen in these mice (7) in contrast to the increased injury in myr-Akt-Tg is consistent with the model that Akt activation is not sufficient for cardioprotection and that PI3K-dependent but Akt-independent signaling plays a critical role in the response to IRI. However, it is not clear why there is no feedback inhibition of IRS-1/PI3K signaling in IGF-1-Tg mice. The potential roles of the level and kinetics of IGF-1 expression remain to be explored, but these results are consistent with adenoviral overexpression studies in cardiomyocytes (data not shown), which also demonstrated paradoxical preservation of IRS-1 protein (in contrast to IGF-1-treated cells). Whatever the mechanism, these findings may have important practical implications for consideration of therapeutic strategies and may underlie our previously unexplained observation that IGF-1 overexpression achieves more effective protection of cardiomyocytes than expression of recombinant protein, even when matched for IGF-1 media levels (47).

Intriguingly, we also observed a reduction in IRS-1 levels in hearts from patients with heart failure. While considerably more and larger samples will be necessary for complete analysis and correlation with Akt activation, we hypothesize that reduced IRS-1 expression represents a feedback mechanism in response to chronic and repetitive stimulation of this signaling pathway by the complex array of cytokines and growth factors increased in heart failure $(48,49)$, many of which are capable of activating IRS/PI3K/Akt signaling. We would anticipate that loss of IRS-1 would produce a relative defect in downstream signaling akin to the insulin resistance seen in type 2 diabetes. A similar blunted response was seen in myr-Akt-Tg hearts during IRI (Supplemental Figure 2). However, in heart failure, in contrast to our mouse model, activation of both Akt and PI3K likely would be increased because of increased stimulation by circulating factors. Reduced IRS-1 levels would blunt the response to these factors, and PI3K/Akt activation would be less than that of comparably stimulated normal hearts.

In summary, here we show that chronic Akt activation in the heart has adverse effects on cardiac injury and functional recovery after IRI through feedback inhibition of upstream signaling pathways. These findings demonstrate that Akt activation alone is not sufficient for cardioprotection and that PI3K-dependent but Aktindependent pathways play a critical role in this setting. Future 
studies will need to be directed at defining the nature and contribution of such pathways to a variety of cardiac pathologies.

\section{Methods}

Animals. The cardiac-specific myr-Akt-Tg mice have been described previously (20). The TG564 line was used for these studies unless otherwise indicated. TG564 mice were backcrossed to C57BL/6 mice for more than 10 generations. dn-Akt-Tg mice were generated analogously through oocyte injection using a cDNA encoding hemagglutinin-tagged dn-Akt (T308A, S473A) (50) under the direction of $\alpha-$ MHC promoter (51). Positive founders were bred to WT C57BL/ 6 mice for 5 generations. Because initial transgene expression was relatively low, hemizygous dn-Akt-Tg mice were crossbred, and mice carrying 2 transgene alleles were selected by quantitative PCR and confirmed by breeding to WT C57BL/6. An overall increase in immunoreactive Akt protein was confirmed in dn-Akt hearts (Supplemental Figure 3). In homozygous dn-Akt mice, cardiac Akt activity and, more modestly, phospho-GSK3 $\beta$ levels were reduced at baseline. Akt kinase activity increased after insulin stimulation, though less than in controls, and these mice were used for the studies described. The IGF-1-Tg (2) and Akt1-null mice (23) have been described previously. Ten- to 15-weekold age-matched mice were used in all experiments, and either littermates or WT mice from lines with at least 5 WT backcrosses (C57BL/6 for myrAkt and dn-Akt mice and FVB/N for IGF-1 mice) were used as NTg controls. All protocols were approved by the Subcommittee on Research Animal Care at Massachusetts General Hospital.

Langendorff model. Mice were heparinized (1,000 IU/kg, i.p.) and anesthetized (pentobarbital, $60 \mathrm{mg} / \mathrm{kg}$, i.p.) and hearts excised and immersed in icecold perfusion buffer. Aortae were cannulated and retrograde-perfused at a constant pressure $(80 \mathrm{mmHg})$ with modified Krebs-Henseleit buffer $(11 \mathrm{mM}$ glucose, $118 \mathrm{mM} \mathrm{NaCl}, 4.7 \mathrm{mM} \mathrm{KCl}, 2.0 \mathrm{mM} \mathrm{CaCl}_{2}, 1.2 \mathrm{mM} \mathrm{MgSO}_{4}, 1.2 \mathrm{mM}$ $\mathrm{KH}_{2} \mathrm{PO}_{4}, 25 \mathrm{mM} \mathrm{NaHCO} 3,0.5 \mathrm{mM}$ EDTA, equilibrated with $95 \% \mathrm{O}_{2} / 5 \%$ $\mathrm{CO}_{2}$ at a $\mathrm{pH}$ of 7.4 ) at $37^{\circ} \mathrm{C}$. A water-filled balloon catheter was introduced into the LV for recording of pressure and heart rate (PowerLab; ADInstruments). The balloon was inflated until a stable LVEDP less than $10 \mathrm{mmHg}$ was obtained. The temperature was maintained at $37^{\circ} \mathrm{C}$ with a water jacket. We monitored coronary flow rate by collecting coronary sinus effluent. No difference in contractile performance was seen between male and female mice, so data from both sexes were combined in all studies.

Ischemia/reperfusion model. For the control samples, hearts were aerobically perfused for 20 minutes. Where indicated, $100 \mathrm{nM}$ insulin was added to the buffer, and hearts were perfused for another 10 minutes. For ischemia/ reperfusion, flow was eliminated for the period indicated after a 20 -minute control perfusion period. Hearts were then reperfused for 40 minutes. Some hearts were treated with either $10 \mathrm{mM} \mathrm{LiCl}$ or $3 \mu \mathrm{M} \mathrm{SB} 21676$ (SigmaAldrich) for 10 minutes before ischemia and during the entire 40-minute reperfusion. Where indicated, wortmannin (Sigma-Aldrich) was added. Subsequently, hearts were snap-frozen in liquid nitrogen and stored at $-80^{\circ} \mathrm{C}$ prior to analysis. For infarct size, triphenyltetrazolium chloride (TTC) staining was performed as described previously (10).

In vivo cardiac function. In vivo hemodynamic analyses were performed by pressure-volume catheterization as described previously (52).

In vivo gene transfer. Recombinant adenovirus $\left(20 \mu \mathrm{l}\right.$ at $1 \times 10^{9}$ particles $\left./ \mathrm{ml}\right)$ encoding myc-tagged BD110 (8) or GFP- $\beta$-galactosidase was injected into the $\mathrm{LV}$ cavity during occlusion of the descending aorta at a body temperature of $18-21^{\circ} \mathrm{C}$, as described previously (52). Three days later, hearts were isolated and subjected to ischemia/reperfusion as described above.

CPK. CPK was measured in the effluent using an enzymatic activity assay developed by the Biochemistry Laboratories at Massachusetts General and Johns Hopkins Hospitals. Values were corrected for coronary flow and heart weight $[\mathrm{CPK}(\mathrm{U} / \mathrm{l}) \times$ coronary flow $(\mathrm{l} / \mathrm{min}) /$ heart wt $(\mathrm{g})=\mathrm{U} / \mathrm{min} / \mathrm{g}]$.
DNA fragmentation. Apoptosis was assessed by DNA fragmentation as described previously (8). The density of the ladder was quantified using NIH Image version 1.63 (http://rsb.info.nih.gov/nih-image/download.html).

Kinase assays. For PI3K, $700 \mu \mathrm{g}$ of total protein was immunoprecipitated with either anti-IRS-1, anti-p110 $\alpha$ (Upstate), anti-p110 $\beta$, or anti-p110 $\gamma$ antibody (Santa Cruz Biotechnology Inc.), and kinase activity was detected by the appearance of radiolabeled ${ }^{32} \mathrm{P}$-labeled phosphatidylinositol 3-phosphate ([32P]PI-3-P) after TLC as described (8). Autoradiographic signals were quantitated using NIH Image version 1.63. Where indicated, PI3K activity was determined by immunoprecipitation with anti-p110 $\alpha$ antibody and a fluorescence polarization assay according to the manufacturer's instructions (Echelon Biosciences Inc.). We determined polarization by measuring the amount of unbound fluorescent probe with a VICTOR3 plate reader (PerkinElmer). Values were normalized to the samples from NTg mice exposed to ischemia/reperfusion. For Akt, the Akt Kinase Assay Kit (Cell Signaling Technology) was used as described previously (20).

Immunoblotting. Immunoblotting was performed as previously described (20) using primary antibodies to IRS-1, p85, phospho-SGK1, SGK1, c-myc (Upstate), phospho-(Ser/Thr) Akt substrate, phospho-Akt (Ser473), Akt, phospho-GSK3 $\beta$ (Ser9) (Cell Signaling Technology), GSK3 (Transduction Laboratories), $\beta$-actin (Sigma-Aldrich), and GAPDH (Abcam Ltd.). Signal was detected using enhanced chemiluminescence, and band intensity was quantified using NIH Image.

Immunoprecipitation. Seven hundred micrograms of total protein from either tissue or cardiomyocytes were immunoprecipitated for $30 \mathrm{~min}$ utes using anti-IRS-1 antibody. Subsequently, Protein A Sepharose beads (Amersham Biosciences) were added for 16 hours. Beads were washed 3 times and proteins solubilized in $2 \times$ SDS sample buffer with boiling followed by immunoblotting using the indicated antibodies.

Detection of proteasomal degradation in vitro. NRCMs were prepared and cultured for 72 hours as described previously (8), prior to incubation in serum-free RPMI1640 for 8 hours before infection with the indicated vectors. After 20 hours, the proteasome inhibitor ALLN (Calbiochem) or the structural control, ALLM (final concentrations, $50 \mu \mathrm{M}$ ), or DMSO alone was added (24). Where indicated, $100 \mathrm{nM}$ wortmannin or $50 \mathrm{nM}$ rapamycin (Sigma-Aldrich) was added. After 30 minutes, insulin (100 nM) was administrated as indicated. After 20 hours, cell lysates were immunoblotted for IRS-1.

Detection of IRS-1 phosphorylation. NRCMs were cotransfected with Ad.IRS-1 and the indicated adenoviral vectors. After 48 hours, cell lysates were immunoprecipitated with anti-IRS-1 antibody or control Ig and then immunoblotted with the indicated antibodies.

Adenoviral vectors. Ad.GFP and Ad.BD110 have been described previously (8). Ad.BD110 encodes myc-tagged BD110, which encodes the PI3K $\mathrm{p} 110 \alpha$ catalytic domain linked to the $\mathrm{p} 85$ regulatory subunit binding domain (25), and an independent GFP expression cassette (8). Both Ad.myr-Akt and Ad.dn-Akt encode separate expression cassettes for the Akt construct and GFP and were constructed by subcloning either myr-Akt or dn-Akt (T308A, S473A; Akt-AA) cDNAs into pAdTrackCMV (Stratagene) and obtaining homologous recombinants through cotransformation with pAdEasy1 (Stratagene) in E. coli BJ5183 (Stratagene) cells as described previously (53). Ad.IRS-1 was kindly provided by K. Ueki (University of Tokyo, Tokyo, Japan).

QRT-PCR analysis. Total RNA was isolated and QRT-PCR performed using the Mx4000 system (Stratagene) as described previously (37). Transcriptional levels were determined using the $\Delta \Delta \mathrm{Ct}$ method with normalization to GAPDH.

Human heart samples. Under a Human Studies Protocol approved by the institutional review board, heart failure samples were obtained from cardiac explants from transplant patients with idiopathic dilated cardiomyopathy. 
Control samples came from unused donor hearts without evidence of cardiac disease. To avoid confounding related to insulin signaling, no samples from diabetic patients were utilized in either group.

Statistical analysis. Data are represented as the mean \pm SEM from at least 3 independent experiments. Student's $t$ test was used to compare the means of normally distributed continuous variables. ANOVA followed by NewmanKeuls post-hoc test for multiple comparisons was used for PI3K assays and in vitro immunoblotting for IRS-1. Two-way ANOVA followed by Bonferroni multiple comparison correction was used for ex vivo LVDP profiles. $P<0.05$ was considered statistically significant.

\section{Acknowledgments}

The authors thank James Flood (Massachusetts General Hospital) for help obtaining CPK determinations, Kohjiro Ueki (University of Tokyo) for the Ad.IRS-1 virus, and Jeffrey Robbins (Cincinnati Children's Hospital Research Foundation) for the $\alpha-\mathrm{MHC}$ promoter.
Work has been supported by grants from the NIH: HL-59521 and a Boston Area Diabetes Research Consortium Pilot Award to A. Rosenzweig; HL-04250 to T. Matsui; HL-67516, RR-016362, and HL-49574 to J.K. Gwathmey; a grant-in-aid from the American Heart Association Northeast Research Consortium to T. Matsui; and a GlaxoSmithKline Fellowship to T. Nagoshi. The Friedrich Miescher Institute is funded by the Novartis Research Foundation.

Received for publication August 18, 2004, and accepted in revised form March 29, 2005.

Address correspondence to: Anthony Rosenzweig, Program in Cardiovascular Gene Therapy, Massachusetts General Hospital, 114 16th Street, Room 2600, Charlestown, Massachusetts 02129, USA. Phone: (617) 726-8286; Fax: (617) 724-7387; E-mail: arosenzweig@partners.org.
1. Buerke, M., et al. 1995. Cardioprotective effect of insulin-like growth factor I in myocardial ischemia followed by reperfusion. Proc. Natl. Acad. Sci. U. S. A. 92:8031-8035.

2. Reiss, K., et al. 1996. Overexpression of insulin-like growth factor- 1 in the heart is coupled with myocyte proliferation in transgenic mice. Proc. Natl. Acad. Sci. U. S. A. 93:8630-8635.

3. Cittadini, A., et al. 1996. Differential cardiac effects of growth hormone and IGF-1 in the rat. Circulation. 93:800-809.

4. Cittadini, A., et al. 1998. Insulin-like growth factor1 but not growth hormone augments mammalian myocardial contractility by sensitizing the myofilament to $\mathrm{Ca} 2+$ through a wortmannin-sensitive pathway. Circ. Res. 83:50-59.

5. Li, Q., et al. 1997. Overexpression of IGF-1 in mice protects from myocyte death after infarction, attenuating ventricular dilation, wall stress, and cardiac hypertrophy. J. Clin. Invest. 100:1991-1999.

6. Li, B., et al. 1999. Insulin-like growth factor-1 attenuates the detrimental impact of nonocclusive coronary artery constriction on the heart. Circ. Res. 84:1007-1019.

7. Yamashita, K., et al. 2001. Reperfusion-activated Akt kinase prevents apoptosis in transgenic mouse hearts overexpressing IGF-1. Circ. Res. 88:609-614.

8. Matsui, T., et al. 1999. Adenoviral gene transfer of activated PI 3-kinase and Akt inhibits apoptosis of hypoxic cardiomyocytes in vitro. Circulation. 100:2373-2379.

9. Fujio, Y., Nguyen, T., Wencker, D., Kitsis, R.N., and Walsh, K. 2000. Akt promotes survival of cardiomyocytes in vitro and protects against ischemiareperfusion injury in mouse heart. Circulation. 101:660-667.

10. Matsui, T., et al. 2001. Akt activation preserves cardiac function and prevents injury after transient cardiac ischemia in vivo. Circulation. 104:330-335.

11. Haq, S., et al. 2001. Differential activation of signal transduction pathways in human hearts with hypertrophy versus advanced heart failure. Circulation. 103:670-677.

12. Rocchi, S., Tartare-Deckert, S., Mothe, I., and Van Obberghen, E. 1995. Identification by mutation of the tyrosine residues in the IRS- 1 affecting association with the tyrosine phosphatase $2 \mathrm{C}$ and phosphatidylinositol 3-kinase. Endocrinology. 136:5291-5297.

13. Araki, E., et al. 1994. Alternative pathway of insulin signalling in mice with targeted disruption of the IRS-1 gene. Nature. 372:186-190.

14. Vanhaesebroeck, B., and Alessi, D.R. 2000. The PI3K-PDK1 connection: more than just a road to PKB. Biochem. J. 346:561-576.

15. Cantley, L.C. 2002. The phosphoinositide 3-kinase pathway. Science. 296:1655-1657.
16. Sugden, P.H. 2002. Signaling pathways activated by vasoactive peptides in the cardiac myocyte and their role in myocardial pathologies. J. Card. Fail. 8(Suppl. 6):S359-S369.

17. Ahmed, N.N., et al. 1993. The proteins encoded by c-akt and v-akt differ in post-translational modification, subcellular localization and oncogenic potential. Oncogene. 8:1957-1963.

18. Webster, M.K., Goya, L., Ge, Y., Maiyar, A.C., and Firestone, G.L. 1993. Characterization of sgk, a novel member of the serine/threonine protein kinase gene family which is transcriptionally induced by glucocorticoids and serum. Mol. Cell. Biol. 13:2031-2040.

19. Aoyama, T., et al. 2005. Serum and glucocorticoidresponsive kinase-1 (SGK1) regulates cardiomyocyte survival and hypertrophic response. Circulation. 111:1652-1659.

20. Matsui, T., et al. 2002. Phenotypic spectrum caused by transgenic overexpression of activated Akt in the heart. J. Biol. Chem. 277:22896-22901.

21. Li, J., DeFea, K., and Roth, R.A. 1999. Modulation of insulin receptor substrate-1 tyrosine phosphorylation by an Akt/PI3-kinase pathway. J. Biol. Chem. 274:9351-9356.

22. Zhande, R., Mitchell, J.J., Wu, J., and Sun, X.J. 2002. Molecular mechanism of insulin-induced degradation of IRS-1. Mol. Cell. Biol. 22:1016-1026.

23. Yang, Z.Z., et al. 2003. Protein kinase B alpha/Akt1 regulates placental development and fetal growth. J. Biol. Chem. 278:32124-32131.

24. Plas, D.R., and Thompson, C.B. 2003. Akt activation promotes degradation of tuberin and FOXO3a via the proteasome. J. Biol. Chem. 278:12361-12366.

25. Kobayashi, M., et al. 1997. Expression of a constitutively active phosphatidylinositol 3-kinase induces process formation in rat PC12 cells. Use of Cre/loxP recombination system. J. Biol. Chem. 272:16089-16092.

26. Giorgino, F., et al. 1999. Islet transplantation restores normal levels of insulin receptor and substrate tyrosine phosphorylation and PI3-kinase activity in skeletal muscle and myocardium of streptozocin-induced diabetic rats. Diabetes. 48:801-812.

27. Desrois, M., et al. 2004. Initial steps of insulin signaling and glucose transport are defective in the type 2 diabetic rat heart. Cardiovasc. Res. 61:288-296.

28. Kim, J.K., et al. 2001. Prevention of fat-induced insulin resistance by salicylate. J. Clin. Invest. 108:437-446. doi:10.1172/JCI200111559.

29. Gao, Z., et al. 2002. Serine phosphorylation of insulin receptor substrate 1 by inhibitor kappa B kinase complex. J. Biol. Chem. 277:48115-48121.

30. Sommerfeld, M.R., Metzger, S., Stosik, M., Ten- nagels, N., and Eckel, J. 2004. In vitro phosphorylation of insulin receptor substrate 1 by protein kinase C-zeta: functional analysis and identification of novel phosphorylation sites. Biochemistry. 43:5888-5901.

31. Moeschel, K., et al. 2004. Protein kinase C-zetainduced phosphorylation of Ser318 in IRS-1 attenuates the interaction with the insulin receptor and the tyrosine phosphorylation of IRS-1.J. Biol. Chem. 279:25157-25163.

32. Aguirre, V., Uchida, T., Yenush, L., Davis, R., and White, M.F. 2000. The c-Jun NH(2)-terminal kinase promotes insulin resistance during association with IRS-1 and phosphorylation of Ser(307). J. Biol. Chem. 275:9047-9054.

33. Haruta, T., et al. 2000. A rapamycin-sensitive pathway down-regulates insulin signaling via phosphorylation and proteasomal degradation of IRS-1. Mol. Endocrinol. 14:783-794.

34. Hirashima, Y., et al. 2003. Insulin down-regulates iIRS-2 expression through the PI3-kinase/Akt pathway. J. Endocrinol. 179:253-266.

35. Zhang, J., et al. 2001. Insulin inhibits transcription of IRS-2 gene in rat liver through an insulin response element (IRE) that resembles IREs of other insulin-repressed genes. Proc. Natl. Acad. Sci. U. S. A. 98:3756-3761.

36. Araki, E., Haag, B.L., 3rd, Matsuda, K., Shichiri, M., and Kahn, C.R. 1995. Characterization and regulation of the mouse insulin receptor substrate gene promoter. Mol. Endocrinol. 9:1367-1379.

37. Cook, S.A., Matsui, T., Li, L., and Rosenzweig, A. 2002. Transcriptional effects of chronic Akt activation in the heart. J. Biol. Chem. 277:22528-22533.

38. Lefer, D.J., et al. 1993. Cardioprotective actions of a monoclonal antibody against CD-18 in myocardial ischemia-reperfusion injury. Circulation. 88:1779-1787.

39. Lefer, D.J., et al. 1997. Peroxynitrite inhibits leukocyte-endothelial cell interactions and protects against ischemia-reperfusion injury in rats. J. Clin. Invest. 99:684-691.

40. Dimmeler, S., et al. 1999. Activation of nitric oxide synthase in endothelial cells by Akt-dependent phosphorylation. Nature. 399:601-605.

41. Fulton, D., et al. 1999. Regulation of endotheliumderived nitric oxide production by the protein kinase Akt. Nature. 399:597-601.

42. Pabla, R., et al. 1996. Nitric oxide attenuates neutrophil-mediated myocardial contractile dysfunction after ischemia and reperfusion. Circ. Res. 78:65-72.

43. Tong, H., Imahashi, K., Steenbergen, C., and Murphy, E. 2002. Phosphorylation of glycogen synthase kinase-3beta during preconditioning through a PI3-kinase-dependent pathway is cardioprotective. 
Circ. Res. 90:377-379.

44. Juhaszova, M., et al. 2004. Glycogen synthase kinase- $3 \beta$ mediates convergence of protection signaling to inhibit the mitochondrial permeability transition pore. J. Clin. Invest. 113:1535-1549. doi:10.1172/JCI200419906.

45. Jonassen, A.K., Sack, M.N., Mjos, O.D., and Yellon, D.M. 2001. Myocardial protection by insulin at reperfusion requires early administration and is mediated via Akt and p70s6 kinase cell-survival signaling. Circ. Res. 89:1191-1198.

46. Tong, H., Chen, W., Steenbergen, C., and Murphy, E. 2000. Ischemic preconditioning activates phos- phatidylinositol-3-kinase upstream of protein kinase C. Circ. Res. 87:309-315.

47. Chao, W., et al. 2003. Strategic advantages of IGF-I expression for cardioprotection. J. Gene Med. 5:277-286.

48. Mann, D.L. 2002. Inflammatory mediators and the failing heart: past, present, and the foreseeable future. Circ. Res. 91:988-998.

49. Dzau, V.J. 1993. Local contractile and growth modulators in the myocardium [review]. Clin. Cardiol. 16(5 Suppl. 2):II5-II9.

50. Kitamura, T., et al. 1998. Requirement for activation of the serine-threonine kinase Akt (protein kinase B) in insulin stimulation of protein synthesis but not of glucose transport. Mol. Cell. Biol. 18:3708-3717.

51. Subramaniam, A., et al. 1991. Tissue-specific regulation of the alpha-myosin heavy chain gene promoter in transgenic mice. J. Biol. Chem. 266:24613-24620.

52. Champion, H.C., et al. 2003. Robust adenoviral and adeno-associated viral gene transfer to the in vivo murine heart: application to study of phospholamban physiology. Circulation. 108:2790-2797.

53. He, T., et al. 1998. A simplified system for generating recombinant adenoviruses. Proc. Natl. Acad. Sci. U. S. A. 95:2509-2514. 